\title{
Remarques à propos de l'évolution des connaissances sur le cerveau*
}

\author{
Par Huldrych M. Koelbing
}

I.

Un homme tombe soudainement par terre sans aucune raison apparente; vous avez causé avec lui, il venait de vous parler d'une manière parfaitement normale; maintenant cependant -

«il perd la voix et étouffe, l'écume lui sort de la bouche, il grince des dents, les mains se tordent, les yeux divergent, toute connaissance est perdue, quelquefois même il y a sortie des excréments» 1 .

Un homme qui est victime d'une telle attaque, n'a-t-il pas été frappé par un dieu ou saisi par un démon? C'est là l'explication qui doit se présenter spontanément à l'esprit du spectateur naïf et terrifié. Après une ou plusieurs minutes, l'état convulsif cesse; peu à peu le malade revient à lui et n'a aucun souvenir du spectacle effrayant qu'il a présenté. Un événement mystérieux, d'apparence surnaturelle - une maladie pas comme les autres - la maladie sacrée. Voici l'opinion courante, et bien compréhensible, qui régnait parmi les Grecs vers 400 avant J.-Chr.

Mais le médecin auquel nous devons la description succinte du «grand mal» épileptique que je viens de vous lire n'est pas de cet avis. Nous n'avons pas de certitude que ce médecin fût Hippocrate de Cos (460-377) lui-même, mais cela reste probable, car le petit traité «De la maladie sacrée» traduit avec une clarté incomparable l'esprit scientifique d'Hippocrate et de son école. L'auteur commence son exposé en affirmant, contrairement à toute évidence $^{2}$ :

«Voici ce qu'il en est de la maladie dite sacrée: elle ne me paraît avoir rien de plus divin ni de plus sacré que les autres, mais la nature et la source en sont les mêmes que pour les autres maladies. Sans doute c'est grâce à l'inexpérience et au merveilleux qu'on en a regardé la nature et la cause comme quelque chose de divin [...].»

Après une polémique acerbe contre «les mages, les purificateurs, les charlatans, les imposteurs», qui prétendent guérir l'épilepsie par des incantations

* Conférence donnée à la Faculté des Sciences de l'Université de Lausanne, le 5 novembre 1984.

Gesnerus 42 (1985) 315-328 
et d'autres moyens magiques, l'auteur se met à démontrer que l'épilepsie a son origine dans le cerveau. Il fait suivre sa démonstration d'une digression sur le rôle du cerveau dans l'organisme. Ce n'est point dans le diaphragme (phrenes), comme nous le lisons chez Homère, que résident l'intelligence et les émotions; c'est le cerveau qui en est responsable ${ }^{3}$ :

«Il faut savoir que, d'une part, les plaisirs, les joies, les ris et les jeux, d'autre part, les chagrins, les peines, les mécontentements et les plaintes ne nous arrivent que du cerveau. C'est par là surtout que nous pensons, comprenons, voyons, entendons, que nous distinguons le laid et le beau, le mal et le bien, l'agréable et le désagréable, et nous les distinguons soit par la coutume, soit par l'utilité qu'elles nous procurent. C'est encore par là que nous sommes fous, que nous délirons, que des craintes et des terreurs nous assiègent, soit la nuit, soit le jour, des insomnies, des erreurs fâcheuses, des soucis sans motifs, des absences de mémoire, des actes inaccoutumés. Tout cela, nous l'éprouvons par le cerveau quand il n'est pas sain, c'est-à-dire quand il est trop chaud, ou trop froid, trop humide ou trop sec, ou quand il a éprouvé quelque autre affection contre nature à laquelle il n'est pas habitué. $[\ldots]$

Pour ces raisons, je regarde le cerveau comme l'organe ayant le plus de puissance dans l'homme, car il nous est, quand il se trouve sain, l'interprète des effets que l'air produit; or l'air lui donne l'intelligence. Les yeux, les oreilles, la langue, les mains, les pieds agissent suivant que le cerveau a de la connaissance; en effet, tout le corps participe à l'intelligence dans la proportion qu'il participe à l'air; or, pour l'intelligence, le cerveau est le messager. Quand l'homme attire en lui le souffle, ce souffle arrive d'abord au cerveau, et c'est de cette façon que l'air se disperse dans le reste du corps, laissant dans le cerveau sa partie la plus active, celle qui est intelligente et connaissante.»

Voici donc le cerveau reconnu par Hippocrate comme le centre directeur de notre vie intellectuelle et morale, le siège de nos perceptions et de nos activités. Il est vrai qu'à peu près un siècle plus tôt le philosophe-médecin Alcméon de Crotone (né vers 540 avant J.-Chr.) avait déjà attribué au cerveau les facultés de la perception et de la réflexion. Mais maintenant cette conception est considérablement approfondie et élargie par Hippocrate, qui inclut dans sa description de l'activité cérébrale les émotions: nos plaisirs et nos joies, nos chagrins et nos peines ont leur source unique dans le cerveau. Et, comme médecin, Hippocrate passe de la psycho-physiologie à la psychopathologie: tout dérangement mental, fût-il même aussi banal qu'un rêve agité, traduit un dérangement cérébral. La conviction des médecins hippocratiques que l'homme tout entier, bien-portant ou malade, reste toujours soumis aux lois de la nature, leur fait concevoir aussi toute la vie psychique, normale ou anormale, comme une fonction cérébrale, donc organique. Les mêmes forces élémentaires du chaud et du froid, du sec et de l'humide, qui par leur déséquilibre causent les maladies du corps, provoquent aussi, par leur 
action sur le cerveau, les maladies de l'âme. C'est là un point de vue rigoureusement somatique, choisi par Hippocrate en vue de développer une théorie naturelle et unitaire des maladies.

La croyance que c'est l'air inspiré - le pneuma, le souffle - qui nous donne la conscience et tout ce qui en dépend, était répandue à l'époque. Vers 430 , alors qu'Hippocrate était un jeune médecin de trente ans, Diogène d'Apollonie énonça l'hypothèse que ce pneuma vivifiant est mélangé dans notre corps au sang, et distribué par les vaisseaux sanguins. De ce point de vue, par conséquent, rien de nouveau dans le traité «De la maladie sacrée».

L'auteur s'en prend cependant à une autre opinion très populaire:

«Quelques-uns disent que nous pensons par le cœur et que cet organe est ce qui éprouve le chagrin et les soucis: il n'en est rien.»

Le cœur est, comme le diaphragme, un organe qui se contracte; il réagit par des contractions plus fortes - nous dirions, par des palpitations - aux émotions de l'âme, mais il n'en est, pour les disciples d'Hippocrate, ni la cause ni le siège.

\section{II.}

La découverte du rôle directeur du cerveau par Alcméon et Hippocrate ne fut pourtant pas une acquisition définitive de la science. Le cerveau fut à nouveau détrôné en faveur du cœur par le plus grand biologiste de l'Antiquité, Aristote (384-322). Pour Aristote, l'âme était le principe de la vie même, la forme qui anime la matière. (Le verbe français animer traduit parfaitement cette pensée aristotélicienne!)

«L'âme est la cause et l'origine du corps vivant»

(esti de hê psychê tou zôntos sômatos aitia kai archê) ${ }^{5}$,

c'est ainsi qu'Aristote définit l'âme dans son traité «De anima», qui est un texte biologique autant que psychologique.

Or, en étudiant le développement du poulet dans l'œuf, Aristote a observé que la première structure qui manifeste une activité vivante est le cœur; avec sa pulsation il apparaît comme une minuscule tâche rouge, pulsante et sautillante - le punctum saliens des Aristotéliciens du Moyen-Age. Il était donc évident que le cœur est la source de la vie dans l'organisme; par conséquent il fallait aussi y localiser l'âme - pour autant que le principe primordial se laisse localiser. 
Le rôle du cerveau n'était cependant pas négligé par Aristote, dont la conviction profonde était que la nature ne fait rien en vain. Le cœur est chaud - et le cerveau était regardé comme froid. De ce contraste Aristote déduisit que le cerveau servait, pour ainsi dire, de contre-poids au cœur: en tempérant un éventuel excès de chaleur du cœur, il maintenait l'équilibre thermique de l'organisme. Voilà une conception caractéristique de l'esprit grec, aspirant toujours à la mesure (to metrion) et au juste milieu (to meson).

Cette interprétation fournit également à Aristote une explication satisfaisante pour le volume extraordinaire du cerveau chez l'homme: de tous les animaux, c'est l'homme qui possède le plus de chaleur; il lui faut aussi, par conséquent, le plus grand cerveau en tant qu'organe refrigérateur!

III.

Au deuxième siècle de notre ère, le médecin Galien de Pergame (130-200) brossa un tableau de l'anatomie et de la physiologie humaines. Il avait beaucoup disséqué - non pas des cadavres humains - mais des animaux morts et vivants, surtout des petits singes de Barbarie. Ceci lui permit de réfuter l'hypothèse aristotélicienne:

«Le toucher indique toujours que l'encéphale est plus chaud que l'air ambiant»,

dit-il dans son œuvre sur «L'utilité des parties du corps». ${ }^{6}$ Comment le cerveau pourrait-il donc refroidir le cœur? Ne serait-il pas plutôt réchauffé, à son tour, par la chaleur émanante du cœur? - En plus:

«Tous les sens sont en rapport avec l'encéphale, s'il faut ajouter foi aux yeux et au tact.» ${ }^{7}$

Galien compte sept paires de nerfs qui prennent leur origine dans le cerveau et le trone cérébral, et qui lient l'encéphale aux organes des sens, mais aussi à certains muscles et viscères. Pour Galien, il est démontré que le cerveau est

«l'organe auquel aboutissent toutes les sensations, où naissent toutes les fantaisies de

l'imagination et toutes les pensées de l'intelligence». ${ }^{8}$

Le cerveau représente

«le principe dirigeant, le siège de l'imagination, de la mémoire et de l'entendement.» ${ }^{9}$

En un mot, le cerveau est «le plus important de tous les organes». ${ }^{10}$

Au $3^{\mathrm{e}}$ siècle avant J.-C., Erasistrate, un des anatomistes éminents d'Alexandrie, qui y avaient pu disséquer des corps humains, avait mis en 
rapport l'aspect hautement différencié de l'encéphale humain avec l'intelligence de l'homme. C'est improbable, dit Galien,

«puisque les ânes mêmes ont un cerveau très-compliqué, tandis que leur caractère imbécile exigerait un encéphale tout à fait simple et sans variété. Il vaut mieux croire que l'intelligence résulte du bon tempérament du corps chargé de penser, quel que soit ce corps, et non de la variété de sa composition.» ${ }^{11}$

Le bon tempérament, cela veut dire l'équilibre des qualités élémentaires, froid et chaud, sec et humide - c'est encore la théorie hippocratique. Mais il y a un facteur auquel Galien attribue encore plus d'importance, l'esprit animal, spiritus animalis:

«C'est $[\ldots]$ à la qualité du pneuma psychique qu'il faut rapporter la perfection de la pensée.» ${ }^{12}$

Rappelons nous ce qu'Hippocrate disait de l'air, qui nous confère l'intelligence! D'Hippocrate à Galien ce concept a évolué: plus de cinq siècles de réflexion philosophique et physiologique d'une part, des connaissances anatomiques beaucoup plus précises de l'autre, imposent une vue différenciée. Je vais essayer d'esquisser succintement la théorie galénique du spiritus animalis, du pneuma psychikon:

L'air que nous inspirons contient un principe vital, indispensable pour le maintien de la vie de notre corps - l'esprit vital (spiritus vitalis, pneuma zôtikon). L'esprit vital passe des poumons à la chambre gauche du cœur; en s'y mêlant à la partie la plus fine du sang veineux, il forme le sang artériel. Tandis que le sang des veines nourrit les organes, le sang des artères les anime et les chauffe. Après être arrivé au cerveau, le sang artériel est, pour ainsi dire, filtré, et l'esprit vital qu'il contient passe des vaisseaux aux ventricules cérébraux. Perfectionné dans les dernières ramifications et sinuosités des artères et dans les ventricules, ce pneuma représente désormais l'esprit animal, le pneuma psychique; nous savons déjà que Galien le tient responsable de l'intelligence. L'esprit animal est l'instrument principal de l'âme ${ }^{13}$. (Plus tard, on parlera volontiers des esprits animaux, au pluriel.) Circulant dans les nerfs, cet esprit transmet au cerveau les impressions sensorielles et permet aux muscles de se contracter.

Ce sont donc les ventricules plutôt que la substance propre du cerveau qui, selon Galien, représentent le siège de l'âme.

En partant de cette théorie, le Moyen-Age est arrivé à une localisation spéculative des facultés mentales, comme le montre une fameuse gravure sur 
bois du $16^{\mathrm{e}}$ siècle $^{14}$ : dans les deux ventricules «antérieurs» (nos ventricules latéraux) résident la perception et l'imagination, dans le troisième la réflexion et le jugement, dans le quatrième la mémoire.

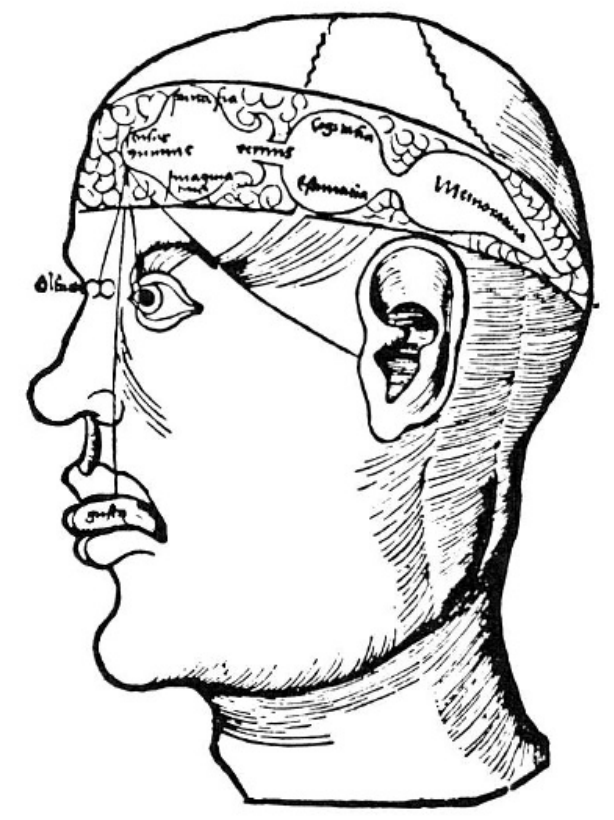

Distribution des facultés de l'âme dans les ventricules («cellules») du cerveau, d'après Gregorius Reisch (1503). Reproduit de E. Clarke et K. Dewhurst,

An Illustrated History of Brain Function, Oxford (Sandfort), 1972

La haute dignité que Galien conférait aux ventricules cérébraux, en tant que lieux de production et réservoirs de l'esprit animal, ne l'empêchait d'ailleurs pas de leur attribuer aussi une fonction de cloaques pour toutes les substances impures ou résiduelles, dont le cerveau devait se débarasser. C'est de là que les rhumes prennent leur origine!

De toute façon, Galien était fier d'avoir démontré

«que l'âme raisonnable habite dans l'encéphale; que nous raisonnons au moyen de ce viscère; que la plus grande partie du pneuma psychique y est renfermée, enfin que ce pneuma acquiert sa propriété spéciale par l'élaboration qu'il y subit.» ${ }^{15}$

Le cerveau n'était cependant pas responsable de nos instincts premiers. Ceux-ci relevaient d'une âme moins différenciée, végétative. Galien lui assignait le foie pour siège.

Voici les connaissances et concepts sur le cerveau, aquis par les Grecs au cours des siècles et finalement revus et systématisés par Galien. Pendant plus 
de mille ans, le système de Galien formera désormais la base apparemment solide de la physiologie et de la médecine. Durant tout le Moyen-Age son défaut majeur restera inaperçu: Galien n’avait fait aucune différence entre les faits observés et leur interprétation théorique et nécessairement souvent hypothétique. Pour ses disciples et successeurs les connaissances, les suppositions et les erreurs de Galien avaient donc le même degré de certitude, voire la même valeur de dogmes infaillibles.

\section{IV.}

«Le siècle rebelle» (the insurgent century) - c'est ainsi que Charles Singer a caractérisé le dix-septième, dans sa brève histoire des idées scientifiques avant $1900^{16}$. La cosmologie et la physique aristotéliciennes furent renversées et remplacées par le système de Copernic (fait public, il est vrai, déjà en 1543), et par la mécanique céleste de Galilée et de Kepler. La révolte se répandit aussi dans les sciences médicales; ici la cible était Galien, dont l'autorité millénaire fut de plus en plus contestée. La découverte de la circulation du sang, publiée en 1628 par William Harvey, porta un coup irrémédiable au système galénique, dans lequel la médecine s'était pourtant installée très confortablement. Dans ce système, tous les principes aussi bien que tous les détails étaient étroitement liés les uns aux autres, sur le plan physiologique aussi bien que sur le plan pathologique et thérapeutique. On ne pouvait donc y introduire une nouvelle conception des mouvements du cœur et du sang sans bouleverser le tout.

En outre, sur le plan méthodologique, la découverte de Harvey représentait un succès éclatant de la pensée mécaniste appliquée à un problème physiologique volontairement isolé. Harvey considérait le sang comme un simple liquide que le cœur pompe à travers le corps dans un circuit perpétuel. Quand le médecin et anatomiste parisien Jean Riolan (fils) lui demandait quelle pourrait être l'utilité de ce prétendu mouvement circulaire du sang, il posait la question primordiale du point de vue galéniste: tout arrangement dans notre organisme devait s'expliquer par son utilité et par sa fin. Mais Harvey répondit que c'était là une question secondaire; l'essentiel était de reconnaître tout d'abord le fait même de la circulation. La recherche de sa fonction dans l'organisme restait encore à faire.

Une fois l'existence de la circulation sanguine admise, il était inévitable que l'on cherchât à élucider et à expliquer d'autres fonctions corporelles 
selon le même principe mécaniste. Le philosophe René Descartes (1596-1650) élabora même la description d'une machine mécanique tout à fait semblable à l'homme ${ }^{17}$. Bien entendu, il ne prétendait pas, dans son «Traité de l'homme», que l'organisme humain, mis à part l'âme rationnelle, n'était qu'un mécanisme. Bien plus subtilement, il partait de la supposition que Dieu pourrait avoir envie de créer un homme artificiel, dont lui, Descartes, allait maintenant décrire le fonctionnement ${ }^{18}$ :

«Ces hommes seront composés, comme nous, d'une âme et d'un corps. [...]

Je suppose que le corps n'est autre chose qu'une statue ou machine de terre, que Dieu forme tout exprès pour la rendre la plus semblable à nous qu'il est possible.»

En terminant sa description, Descartes affirme ${ }^{19}$ :

«Je désire [...] que vous considériez que ces fonctions [c'est-à-dire toutes les fonctions végétatives, animales et même psychiques, à l'exception du raisonnement] suivent toutes naturellement, en cette machine, de la seule disposition de ses organes, ne plus ne moins que font les mouvements d'une horloge, ou autre automate, de celle de ses contrepoids et de ses roues; en sorte qu'il ne faut point à leur occasion concevoir en elle aucune autre âme végétative, ni sensitive, ni aucun autre principe de mouvement et de vie, que son sang et ses esprits, agités par la chaleur du feu qui brûle continuellement dans son cœur, et qui n'est point d'autre nature que tous les feux qui sont dans les corps inanimés.»

«Le sang et les esprits» - voici encore deux notions fondamentales de la physiologie galénique. Mais les «esprits animaux» de Descartes sont de nature franchement matérielle et corpusculaire. Ils sont formés par les corpuscules les plus subtils du sang, qui sortent des petites artères du cerveau en passant par les pores des vaisseaux vers la structure qui occupe le centre même du cerveau - la glande pinéale. De là, les esprits sont dirigés, à l'intérieur des nerfs, vers tous les muscles du corps.

Comment la glande pinéale accède-t-elle, dans la pensée de Descartes, au rôle de centre directeur du cerveau? (C'est là, aussi, que l'âme rationnelle entrera en contact avec la machine du corps!) La raison en est essentiellement géométrique: la glande pinéale se trouve située au centre de l'encéphale et en ligne droite au-dessus du cœur; dans le courant chaud du sang artériel, qui monte du cœur, les corpuscules les plus légers y arriveront donc tout naturellement par la voie la plus courte...

Il est évident que Descartes n'a pas disséqué lui-même un cerveau, puisqu'il suppose que la glande pinéale peut se pencher d'un côté ou de l'autre suivant l'afflux des esprits ou des stimuli sensoriels. En changeant sa 
position de la sorte, elle dirige à son tour les esprits moteurs vers les différentes parties du corps.

Il y a donc, dans la physiologie du $17^{\mathrm{e}}$ siècle, non seulement le raisonnement mécaniste qui s'applique plus ou moins fructueusement à la recherche, mais encore une spéculation mécaniste qui risque de perdre tout contact avec la réalité. Presqu'immédiatement après la publication posthume du «Traité de l'homme» de Descartes, l'anatomiste danois Niels Stensen (Nicolaus Steno) fit à Paris son «Discours sur le cerveau». Dans ce discours, il montre que pour arriver à une meilleure connaissance du cerveau, il faut procéder non pas par la spéculation théorique, mais par l'étude concrète, anatomique de l'organe réel. ${ }^{20}$

Reste encore à savoir quel degré de réalité Descartes lui-même assignait à la description de son homme mécanique. Son intention proclamée qu'il allait décrire quelque chose de fictif (mais que Dieu pourrait créer s'il le voulait) le mettait en tout cas à l'abri de toute accusation d'hérésie. Mais vers la fin de son exposé il exprime sa conviction qu'il serait difficile d'expliquer d'une manière plus simple, et par conséquent plus plausible, le fonctionnement de notre organisme tel qu'il existe ${ }^{21}$.

L'homme et son cerveau - des machines? Des concepts à la manière de la machine humaine de Descartes n'ont cessé de se suivre depuis. La comparaison la plus moderne est celle du cerveau et de l'ordinateur. Dans ces comparaisons tout dépend, à mon avis, de la valeur qu'on leur donne. Si l'on reste conscient qu'il s'agit d'analogies, elles peuvent mieux nous faire comprendre comme nous sommes faits et comme la vie intérieure de notre organisme est réglée. Si, d'autre part, nous voudrions établir une identité réelle entre homme et machine, en disant que l'homme ou le cerveau n'est qu'une machine extrèmement compliquée, alors nous nous éloignerions d'une vraie connaissance de nous-même.

V.

Il ressort de ce que je viens de dire que jusqu'au $17^{\mathrm{e}}$ siècle la fonction $\mathrm{du}$ cerveau a toujours été envisagée d'une manière globale. Les investigateurs du $18^{\mathrm{e}}$ et surtout ceux du $19^{\mathrm{e}}$ siècle ont pénétré, de plus en plus, dans les détails, ce qui leur a permis d'arriver à des connaissances plus valides.

Par exemple, les expériences de Marie-Jean-Pierre Flourens (17941864), faites dans les années vingt et trente du $19^{\mathrm{e}}$ siècle sur des espèces 
variées de vertébrés, révélèrent des différences fondamentales entre les fonctions de différentes parties de l'encéphale. Si l'on touche un certain point dans le bulbe cérébral, l'animal ne respire plus; Flourens nomma ce point notre centre respiratoire - le nœud vital (1837). Le cerveau proprement dit das Großhirn - se trouva être le siège exclusif des facultés du mouvement volontaire, de la perception sensorielle et, par conséquent, de l'intelligence. Quant au cervelet ( Kleinhirn), Flourens démontra sa fonction coordinatrice pour les mouvements du corps.

Les investigations de localisation fonctionnelle ont été poursuivies depuis à l'aide de méthodes de plus en plus différenciées et raffinées, jusqu'à la stimulation ponctuelle par des micro-électrodes (voir plus loin). La découverte du centre moteur du langage articulé dans la troisième circonvolution frontale de l'hémisphère gauche, en 1861, est due à Paul Broca (1824-1890), chirurgien et anthropologiste. Elle a résulté de l'examen du cerveau d'un malade qui, de son vivant, avait souffert d'aphasie motrice ${ }^{22}$.

Nombreuses étaient au $19^{\mathrm{e}}$ siècle les tentatives de mettre en rapport les maladies mentales avec des altérations anatomiques et spécifiques du cerveau. En effet, il ne manquait pas de publications à ce sujet, mais les résultats de ces études anatomiques, voire histologiques, étaient pour la plupart trompeurs; le psychiatre et neurologue Franz Nissl (1860-1919) les classa comme «mythologie du cerveau» (Hirnmythologie). Pour les maladies mentales, la médecine moderne n'a pas réussi à réaliser la vision d'Hippocrate, qui en cherchait les causes dans des altérations tangibles du cerveau!

A cet égard, un passage du traité des maladies mentales de Wilhelm Griesinger (1817-1868) mérite d'être retenu ${ }^{23}$. Tout au début de son livre, Griesinger déclare que les maladies psychiques sont essentiellement des maladies du cerveau. Mais un peu plus loin, il nous rappelle que toutes les connaissances sur les processus biochimiques, bio-électriques ou autres ne sauraient nous faire comprendre comment les activités somatiques, les changements matériels qui se déroulent dans le cerveau, peuvent donner naissance à des réalités d'ordre psychologique:

«Wirkliche Auskunft über das Geschehen in der Seele vermag weder der Materialismus zu geben, der die Seelenvorgänge aus den körperlichen, noch der Spiritualismus, der den Leib aus der Seele erklären will. Wüßten wir auch alles, was im Gehirn bei seiner Tätigkeit vorgeht, könnten wir alle chemischen, elektrischen etc. Prozesse bis in ihr letztes Detail durchschauen - was nützte es?

«Alle Schwingungen und Vibrationen, alles Elektrische und Mechanische ist doch immer noch kein Seelenzustand, kein Vorstellen. Wie es zu diesem werden kann - dies Rätsel wird 
wohl ungelöst bleiben bis ans Ende der Zeiten, und ich glaube, wenn heute ein Engel vom Himmel käme und uns alles erklärte, unser Verstand wäre gar nicht fähig, es nur zu begreifen!»

C'est que l'instrument qui nous sert à comprendre ce qui se passe dans notre cerveau n'est autre que ce cerveau même!

Mis à part les interprétations arbitraires de données histologiques, l'étude microscopique du cerveau était naturellement d'une très grande valeur. Mais il est intéressant de constater que le fondateur de la pathologie cellulaire, Rudolf Virchow (1821-1902), s'y référait (en 1855/58) pour mettre en doute le rôle directeur du cerveau dans l'organisme ${ }^{24}$. Démocrate de 1848, Virchow prenait aussi l'organisme pour

«un Etat libre composé d'individus égaux en droits, bien qu'inégaux en aptitudes, qui tient ensemble parce que les uns dépendent des autres et parce qu'il existe certains centres d'organisation».

Sur le plan scientifique, Virchow luttait pour les droits du «tiers-état du grand nombre des petits éléments» - les cellulles - contre «l'aristocratie et l'hiérarchie du sang et des nerfs». Il ne voulait surtout pas d'hiérarchie nerveuse en pathologie, et il ne croyait pas que des influences nerveuses pouvaient rendre malades d'autres organes. Il était donc logique qu'il contesta au cerveau le privilège de représenter et de garantir l'unité de l'organisme ${ }^{25}$ :

«Même si l'on ne dispose que d'idées très sommaires sur l'anatomie des nerfs, on ne devrait pas se cacher que cette unité [du système nerveux] est des plus précaires. Le seul scalpel montre déjà l'appareil nerveux comme un système composé d'une multitude extraordinaire de particules plus ou moins équivalentes, sans centre reconnaissable. Les éléments se multiplient d'autant plus que nous les examinons plus exactement au microscope. [...] un nombre infini d'éléments cellulaires relativement autonomes et pour la plupart indépendants les uns des autres font leur apparition.»

Tout en poursuivant l'investigation minutieuse de sa structure et de ses fonctions, les neurophysiologistes du $20^{\mathrm{e}}$ siècle ont rétabli le cerveau dans sa dignité de garant de l'unité, de la Ganzheit, de notre organisme. J'aimerais encore mentionner deux personnalités: l'Anglais Charles Scott Sherrington et le Suisse Walter Rudolf Hess. L'œuvre de chacun d'eux a été couronnée par le Prix Nobel, respectivement en 1932 et en 1949.

Sherrington (1857-1952), homme d'une grande culture (et d'ailleurs aussi pionnier du ski dans l'Oberland Bernois, à Grindelwald), était successivement professeur de physiologie à Londres, à Liverpool et à Oxford. Nous lui devons le terme de synapse; avant de proposer, vers 1900, ce nom pour les 
points de contact entre deux neurones, il consultait prudemment un ami professeur de philologie classique. Il s'efforçait, selon Fulton, de traduire les données anatomiques en langage physiologique. Et les amas désordonnés de cellules cérébrales, qui avaient tant impressionné Virchow, aussi bien que les masses des fibres nerveuses commençaient à lui révéler leur ordre discret, mais plein de sens. Le Prix Nobel fut décerné à Sherrington pour ses travaux sur les neurones, mais il envisageait la fonction de ces éléments nerveux dans l'ensemble d'une activité coordonnée et intégrante. En 1906 déjà parut son fameux livre de synthèse, "The Integrative Action of the Nervous System" (New Haven, Yale University Press). Il démontra notamment que dans l'activité du système nerveux l'inhibition est tout aussi importante que l'excitation, et que dans le cerveau il y a toujours coopération harmonieuse de ces deux facteurs.

Hess (1881-1973) exerçait d'abord l'ophtalmologie à Rapperswil, au bord du lac de Zurich, parce qu'il était obligé de nourrir sa famille. En 1917, le Conseil d'Etat du Canton de Zurich l'appelait à la chaire de physiologie, contrairement à l'avis de la Faculté de Médecine, qui aurait préféré à ce jeune homme peu connu, un physiologiste réputé d'Allemagne. Hess enseignait la physiologie à l'Université de Zürich jusqu'en 1951, tout en étudiant le comportement de ses chats de laboratoire en stimulant des points précis dans leur diencéphale par le moyen de micro-électrodes. Une fois implantées, ces micro-électrodes ne gênaient pas les animaux dans leur vie ordinaire. Mais la stimulation ponctuelle des centres diencéphaliques changeait entièrement leur comportement; ils pouvaient, p.ex., s'endormir ou, au contraire, montrer une attitude agressive. Au cours des années, Hess reconnut qu'à son niveau le plus élevé, le système nerveux végétatif est organisé de manière à régler l'état de l'organisme tout entier. Soit que celui-ci soit mis dans un état propre à se défendre et à se maintenir dans le monde extérieur: c'est l'at titude ergotrope. Soit qu'il soit disposé au repos et à la régénération: c'est l'état trophotrope. Ainsi le rôle coordinateur et directeur du cerveau pour les manifestations vitales de l'organisme a été mis en relief par Hess d'une manière admirable ${ }^{26}$. Ses micro-électrodes sont d'ailleurs devenues de précieux instruments de microchirurgie thérapeutique.

Un tout dernier point: Les recherches des dernières décennies ont montré que la régulation nerveuse de notre organisme, effectuée par le cerveau, est intimement liée à un autre grand système de régulation, celui des hormones. En ce qui concerne les deux glandes attachées à l'encéphale, nous constatons du point de vue historique une évolution curieuse: 
- La glande pinéale, dont Descartes avait fait le centre même de toute l'activité cérébrale, voire le véritable siège de l'âme, ne joue plus qu'un rôle insignifiant dans la nouvelle neuro-endocrinologie.

- L'hypophyse, d'autre part, décrite par Galien comme un simple filtre pour les déchets du cerveau ${ }^{27}$, est devenue l'organe qui, pour ainsi dire, dirige la grande symphonie hormonale de notre corps.

La recherche sur le cerveau et ses annexes fut riche en surprises; elle le restera certainement.

\section{Notes bibliographiques}

${ }^{1}$ Collection Hippocratique, De la maladie sacrée, ch. 7. In CEuvres complètes d'Hippocrate, vol.6, édition Littré, E., Paris (Baillière), 1849, p. 373.

2 Op. cit., Littré 6, p.353. - Joly, R., Hippocrate - médecine grecque, Paris (Gallimard), 1964, p. 88 .

${ }^{3}$ Op. cit., Littré 6, p. 387-391. - Joly, R., p. 103-105.

4 Op. cit., Littré 6, p. 393. - Joly, R., p. 106.

${ }^{5}$ Aristote, De anima, livre 2, ch. 4, 415 b.

${ }^{6}$ Galien, De usu partium/De l'Utilité des parties du corps humain, livre 8, ch.3, cité d'après Daremberg, Ch., CEuvres anatomiques, physiologiques et médicales de Galien, tome 1, Paris (Baillière), 1854, p. 531 .

7 Ibid., p. 534 .

${ }^{8}$ Ibid., ch. 7, p. 541

${ }^{9}$ Ibid., p. 544 .

${ }^{10}$ Ibid., livre 9, ch. 4, p. 578.

11 Ibid., livre 8, ch. 13, p. 563.

12 Ibid.

${ }^{13}$ Galien, De placitis Hippocratis et Platonis, livre 7, éd. Kühn, C.G., vol.5, p.606s.

14 Reisch, G., Margarita philosophica, Freiburg i. Br. (Schott) 1503.

${ }^{15}$ Galien, De usu partium, livre 9, ch.4, p. 577.

${ }^{16}$ Singer, Ch., A Short History of Scientific Ideas to 1900, Oxford (Clarendon) 1959.

${ }^{17}$ L'homme, de René Descartes [...], Paris (Le Gras), 1664. La traduction latine parut déjà en 1662, à Leyde, chez Leffen \& Moyard.

${ }^{18}$ Cité d'après Adam, Ch. et Tannery, P. (éd.s), Euvres de Descartes, vol.11, Paris (Vrin) 1967, p.119s. Orthographe modernisé.

19 Ibid., p. 202.

${ }^{20}$ Faller, A., Wertschätzung von Stensens «Discours sur l'anatomie du cerveau» im Verlaufe von drei Jahrhunderten. Publication de la Société suisse d'histoire de la médecine et des sciences naturelles, fasc.35, Aarau/Frankfurt a.M./Salzburg (Sauerländer) 1981.

${ }^{21}$ Descartes, R., op.cit. (note 17/18), p. 201.

${ }^{22}$ Broca P. P., «Remarques sur le siège de la faculté du langage articulé, suivies d'une observation d'aphémie (perte de la parole)». Bull.Soc. anat. Paris 36 (1861), p.330-357. 
${ }^{23}$ Griesinger, W., Die Pathologie und Therapie der psychischen Krankheiten, 4. Aufl. (unveränderter Nachdruck der 2. Aufl., Stuttgart 1861), Braunschweig (Wreden) 1876, p.6.

${ }^{24}$ Voir Koelbing, H.M., «Rudolf Virchow und die moderne Pathologie». Münch.med. Wochenschrift 110 (1968), p. 349-354.

${ }^{25}$ Virchow, R., Die Cellularpathologie in ihrer Begründung auf physiologische und pathologische Gewebelehre, Berlin (Hirschwald) 1858, p.204s.

${ }^{26}$ Voir l'œuvre de synthèse de Hess, W.R., Die funktionelle Organisation des vegetativen Nervensystems, Basel (Schwabe) 1948, p. 226.

${ }^{27}$ Galien, De usu partium, livre 9, ch.3, éd. Daremberg (voir note 6), tome 1, p.574s.

\section{Summary}

Remarks upon the evolution of our knowledge of the brain

The following steps are discussed:

1. Hippocratic medicine: the brain is the center of psychic activity and the seat of mental disorders including epilepsy.

2. Galen and Galenists: elaboration of this concept; the pneuma (spiritus animalis) is the instrument of sensation, motion and thinking; mental faculties are located in the cerebral ventricles.

3. Descartes: a mechanic model of brain and nerve function. Man as a machine.

4. Complementary aspects of cerebral activity in 19th/20th century physiology: localisation of special functions - coordinative action of the brain for the benefit of the whole organism (Flourens, Broca, Virchow-Griesinger, Sherrington, Hess).

Prof. Dr. med. Huldrych M. Koelbing

Vorsteher des Medizinhistorischen Institutes der Universität Zürich

Rämistraße 71

CH-8006 Zürich 